\title{
Collaboration System Design of the Transportation Platform
}

\author{
Zhongwen Wang*, Dong Liang, Ruizhen Duan, and Mingshan Chi \\ Rongcheng College, Harbin University of Science and Technology, Rongcheng, 264300, China
}

\begin{abstract}
In order to reduce the labor intensity of workers in automatic production lines handling material, an Automated Guided Vehicle collaboration system (AGV) is designed in this paper. On the basis of analyzing the differential steering principle, the control strategy of the AGV tracking and the automatic tracking control are designed based on the fuzzy algorithm and PID algorithm respectively, so as to realize the control of PC for the transport of goods. Additionally, the PC control software is specially designed for the system. This system is tested on the simulation and experiment environment and the results show that the AGV has the advantages of high guidance precision, following stability and high reliability. The fleet operation can effectively improve the work efficiency and reduce labor costs.
\end{abstract}

Keywords: AGV; fuzzy control; PID control; remote control; AGV scheduling

(Submitted on November 2, 2018; Revised on December 5, 2018; Accepted on January 6, 2019)

(C) 2019 Totem Publisher, Inc. All rights reserved.

\section{Introduction}

With the development of modern industrial technology, the enterprises pay much attention to the automaticity of the production process to improve the efficiency of production and ensure the product quality [1-3]. The transportations of heavy tasks or workpieces often exist in industrial production, which costs a lot of labor [4-5]. The application of the AGV can significantly reduce the amount of labor and improve the efficiency of work [6-7]. It is widely used in industrial production, logistics, military, and hospital drug management [8-9]. However, the single AGV has many shortcomings in handling a large number of tasks. The collaboration of many vehicles is better at improving the work efficiency. This paper describes in detail the overall design process of the AGV collaborative system, which is characterized by high efficiency, intelligence and low cost. It mainly adopted the PC to control the collaboration of the AGV to complete the fundamental transport task efficiently.

\section{Design Scheme of the System}

The collaboration System of the intelligent Transportation Platform mainly contains the PC control software and multiple AGVs. The PC control software can monitor real-time information of each AGV and then control it. When multiple AGVs form a run, they also depend on the PC control software to schedule uniformly. With the control system and other modules, the AGV itself can work properly in their cooperation. The PC control software and the AGV are connected through the wireless network to transfer the control information. The overall structure of the system is shown in Figure 1.

\section{Method Overall Scheme Design of AGV}

\subsection{Overall Structure}

The modular design idea is applied in the structure design process of the AGV, which is convenient for the disassembling and replacement of parts. The layout of the whole structure is mainly based on the function of the related modules. The main control board used to control the AGV movement is installed in the AGV inside. The tracking sensor used to detect the road information is installed at the bottom of the AGV with the same distance interval. The distance detection device is

* Corresponding author.

E-mail address: wangzhongwen1979@qq.com 
placed in the front of the AGV with the purpose to detect the obstacles. The power system contains the power supply, motor and the transmission system, which are installed at the bottom. In addition, the AGV also contains the wireless communication device with the PC, light hint device, etc. The overall structure of the AGV model is shown in Figure 2.
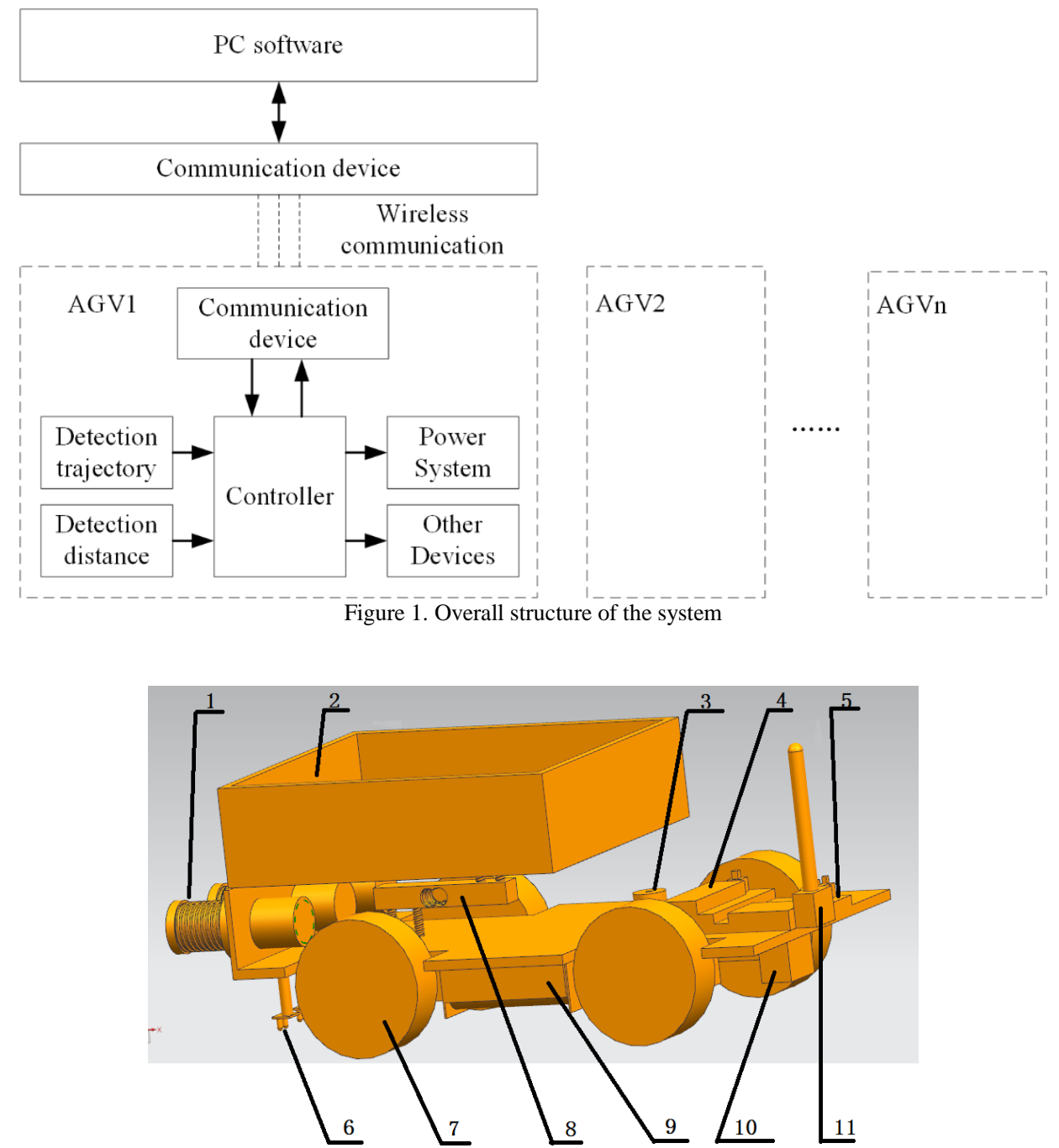

1. Distance sensor; 2. The weighing platform; 3. Alarm device; 4. Main control panel; 5. Switch; 6. Tracking sensor;

7. Wheel; 8. Weighing sensor; 9. Power system; 10. Motor driven board; 11. Communication device

Figure 2. The overall structure of the AGV

\subsection{Design of Steering System}

The AGV dynamic system adopts the battery as the power supply. The choice of the motor as the driving device has the advantages of fast response and simple control. In order to guarantee the compact structure of the AGV, the power system chooses the differential steering design.

By adjusting the rotational speed steering of the wheelchair on both sides, the car can realize the motion along different paths. As shown in Figure 3, the dotted line is the car body's straight trajectory, solid lines are the speed difference actually driving curve track along a straight line, the velocity of the left and right side of the wheel are $V_{l}$ and $V_{r}$ respectively, and the wheel span is $w[10]$.

During the motion time $t$, the actual distance of the left wheel is:

$$
D_{l}=V_{l} \times t
$$

The actual distance of the right wheel is: 


$$
D_{r}=V_{r} \times t
$$

So, the distance difference of the right and left wheels is calculated below:

$$
\Delta D=D_{l}-D_{r}=\left(V_{l}-V_{r}\right) \times t
$$

When the time $t$ is as small as possible, the arc $\Delta D$ can be seen as a straight line, so the angle of the body axis with the linear trajectory must satisfy the following equations:

$$
\tan \theta=\Delta D / w
$$

$$
\theta=\arctan \frac{\left(V_{l}-V_{r}\right) \cdot t}{w}
$$

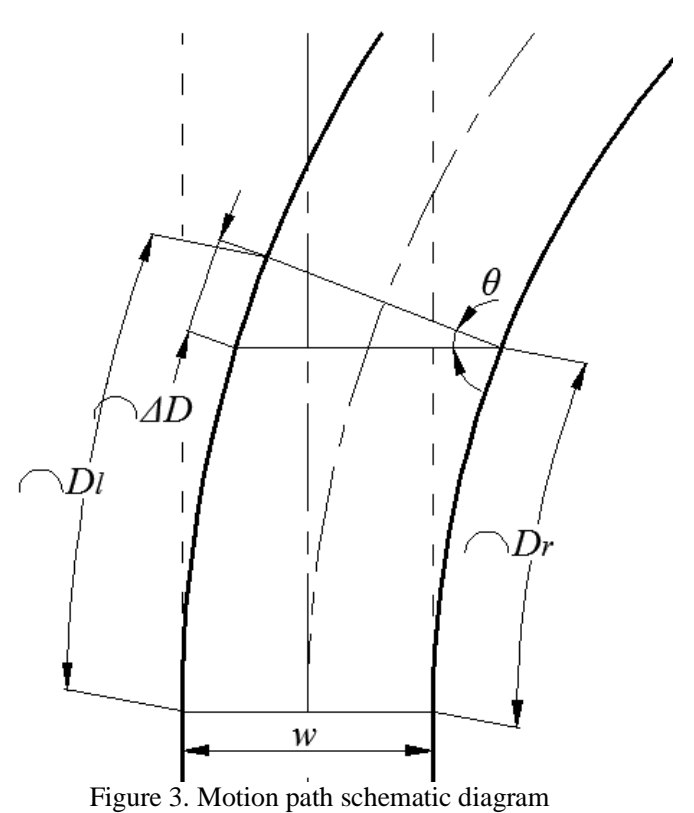

So, we can obtain different angles by changing the speed difference of the wheelchair on both sides. When the velocity is $V_{l}>V_{r}$, the car turns right; $V_{l}<V_{r}$, the car turns left; $V_{l}=V_{r}$, the car moves straight.

\subsection{Tracking Control based on Fuzzy Algorithm}

The highly nonlinear problems exist in the AGV direction control and speed control. However, the AGV requires good realtime performance. So, it is difficult to establish an accurate mathematical model. The traditional control method depended on the precise mathematical model of the control process. This method has poor robustness and may not be stable in some systems with complex dynamic characteristics, so the control effect is not good. In this paper, the fuzzy control is used to control the motion direction of the AGV. This is a kind of intelligent control method, which has the advantages of fast response and smaller overshoot volume [11-13].

By detecting the road information with a tracking sensor, the AGV judges the car position relative to the pre-selected orbit. The tracking sensors are in the front of the car body with the same distance interval, shown in Figure 4.

There are direction deviation $\alpha$ and position deviation $l$ between the body axis and the central line of the orbit. In order to simplify the control process, all the deviations are collectively referred to as deviation $e$, the rate of change is $e c$, $e c=e_{i}-e_{i-1}$. 


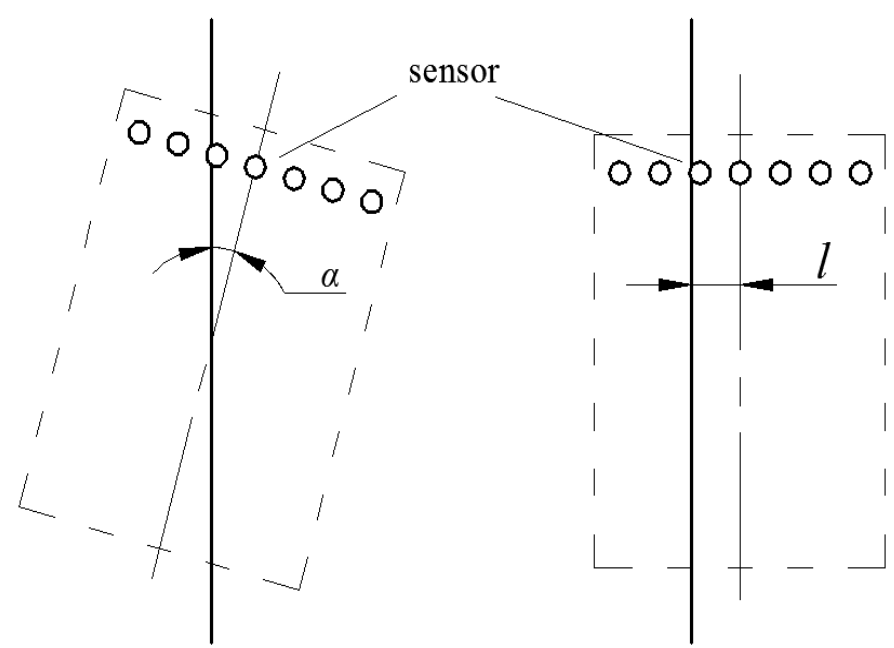

Figure 4. Body deflection diagram

Code the body deviation $e$ of the sensor, recording each sensor's detection status as $J_{1}, J_{2}, J_{3}, J_{4}, J_{5}, J_{6}, J_{7}$, respectively. When the sensor tracks the orbit, the value is 1 , otherwise 0 .

$$
e=\frac{\sum_{i=1}^{7} a_{i} J_{i}}{\sum J},\left\{\begin{array}{l}
a_{1}=-a_{7}=-4 \\
a_{2}=-a_{6}=-2 \\
a_{3}=-a_{5}=-1 \\
a_{4}=0
\end{array}\right.
$$

After each detecting of the sensor, the new deviation $e=\{-4,-3,-2,-1.5,-1,-0.5,0,0.5,1,1.5,2,3,4\}$ can be calculated. The deviation change rate $e c$ can also be obtained according to the basic domain $\{-3,-2,-1,0,1,2,3\}$ that was established with these values.

During the fuzzy processing, we set the fuzzy value of $e$ as $E$, the fuzzy value of $e c$ as $E C$ and $U$ as the variable value of direction control value. Additionally, we select seven kinds of linguistic value: NB, NM, NS, ZO, PS, PM, PB, which respectively mean negative big, negative medium, negative small, zero, positive small, positive medium and positive big, to form the seven fuzzy subsets [14-15].

The fuzzy controller is a dual input and single output fuzzy controller, adopting the fuzzy conditional statement of "if $E$ and $E C$ then $U$ ". According to the analysis of the road and the experimental testing data, the rules of establishing fuzzy control are as shown in Table 1. The degree of membership function of output $U$ is shown in Figure 5.

Table 1. Fuzzy control rules

\begin{tabular}{|c|c|c|c|c|c|c|c|c|}
\hline & \multirow{2}{*}{$J$} & \multicolumn{7}{|c|}{$E C$} \\
\hline & & NB & NM & NS & $\mathrm{ZO}$ & PS & $\mathrm{PM}$ & $\mathrm{PB}$ \\
\hline \multirow{7}{*}{$E$} & NB & PB & PB & PB & PB & PM & $\mathrm{ZO}$ & $\mathrm{ZO}$ \\
\hline & NM & PB & PB & PB & PM & PM & $\mathrm{ZO}$ & $\mathrm{ZO}$ \\
\hline & NS & PB & PM & PM & PS & $\mathrm{ZO}$ & NS & $\mathrm{NM}$ \\
\hline & $\mathrm{ZO}$ & PM & PM & PS & $\mathrm{ZO}$ & NS & $\mathrm{NM}$ & $\mathrm{NM}$ \\
\hline & PS & PS & PS & $\mathrm{ZO}$ & NM & NM & $\mathrm{NM}$ & NB \\
\hline & PM & $\mathrm{ZO}$ & $\mathrm{ZO}$ & $\mathrm{ZO}$ & NM & NB & NB & NB \\
\hline & PB & $\mathrm{ZO}$ & NS & $\mathrm{NB}$ & NB & NB & NB & NB \\
\hline
\end{tabular}

The exact solution value $\Delta V$ can be obtained from the reverse solution. We can adjust the current value $V_{l}, V_{r}$, and change the speed difference of the left and right side of wheel to complete the tracking control. The controller structure is shown in Figure 6. 


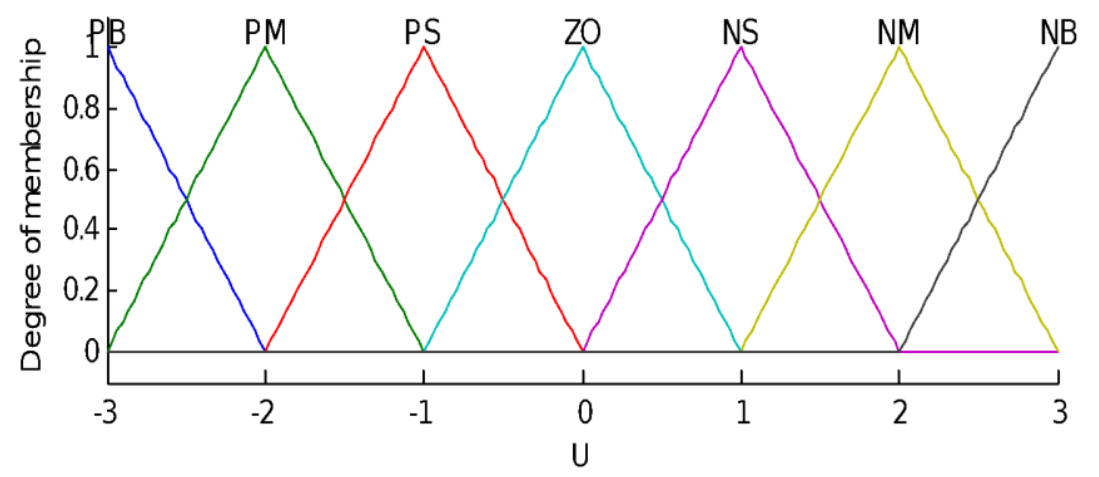

Figure 5. Membership function of $U$

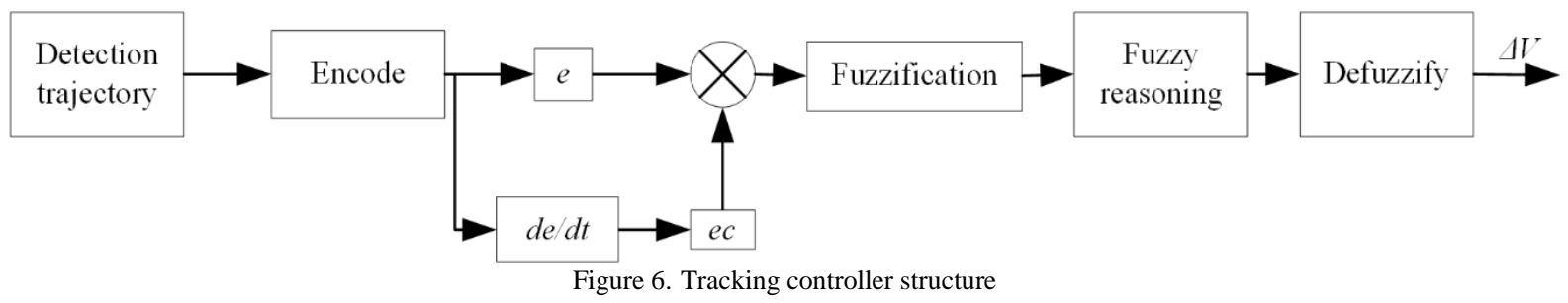

\subsection{Automatic Tracking Control based on PID Algorithm}

The AGV has to keep a safe distance with the front or behind car in the working process. In this design, the distance sensor fixed in the front is used to detect the distance with the front AGV to regulate the speed of the vehicle. On the premise that the distances with the front and rear vehicle are the constant, make the vehicle move smoothly as far as possible.

By the integral relationship of the speed and distance, the distance control can be converted to simple speed control. In a closed loop of a DC motor speed control system, the PID control with the characters of simple structure and stable performance is often chosen. If joining the corresponding parameter self-turning, the accuracy and dynamic of the PID can be improved [16-19].

Choose the velocity of the vehicle axis as the velocity of the vehicle, $V_{c}=\frac{V_{l}+V_{r}}{2}$.

Assume the speed of the front vehicle is $V_{c f}$ and the speed of the rear vehicle is $V_{c b}$. During the running time $T$, the distance of the two cars is calculated in Equation (7).

$$
d=\int_{0}^{T}\left(V_{c f}-V_{c b}\right) \mathrm{d} T
$$

It can easily find that the distance $d$ is related to the value of $V_{c f}-V_{c b}$. Considering that the control system of the transport platform cannot obtain the velocity of the front vehicle, we suppose the $V_{c f}$ and the distance $d$ both are constant during the running when justly changing the speed $V_{c b}$ of the behind vehicle. When the distance $d$ is changing bigger, increasing the speed $V_{c b}$ can shorten the distance $d$ and vice versa.

Select the vehicle distance $d$ as the input value of the PID control system. The output is the velocity $V_{c b}$ of the rear vehicle. The structure of the complex controller composed of the fuzzy control and PID control is shown in Figure 7.

\section{Design of the PC Control Software}

\subsection{Function Requirements}

PC control software is the core of the whole collaborative system, with the following features. 
1) Monitor the operating status of each AGV;

2) Unified schedule for the formation of multiple AGV during automatic running;

3) Display and store the quality information of goods;

4) Help the staffs to remote control AGV.

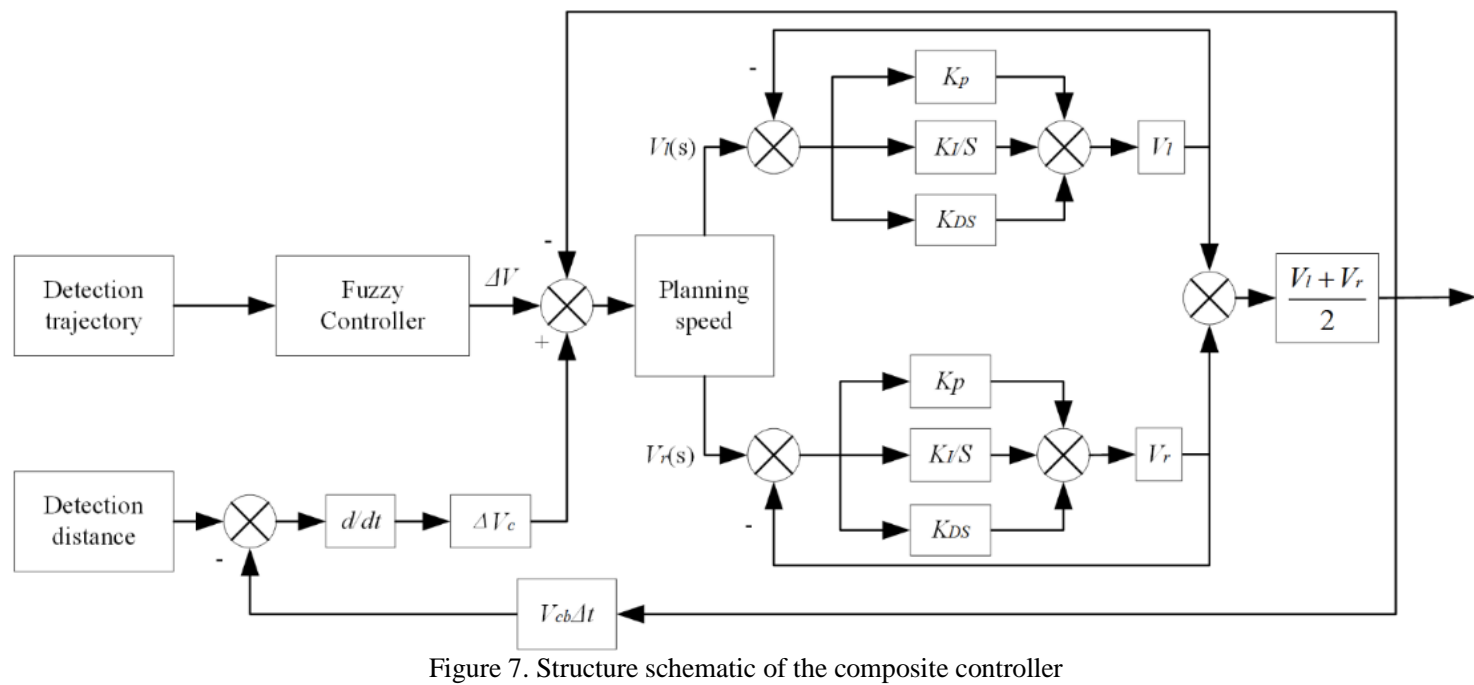

\subsection{Communication Protocol}

In order to meet the requirement of real-time monitoring, the PC control software and AGVs should have continuous communication connection. So, this paper designs the communication protocol between PC control software and AGV.

1) The instruction format of PC control software is as follows.

2) The sign of beginning + Number of AGV + Control instruction + The sign of end.

3) The return instruction format of $\mathrm{AGV}$ is as follows.

4) The sign of beginning + Number of $\mathrm{AGV}+$ Weight of cargo + running status + The sign of end.

\subsection{Communication Connection}

The control software of the PC can monitor the multiple AGVs simultaneously. The frequency of sending and receiving data is low and the amount of data is small. There is no communication connection between AGVs, so there is no need for the high-speed network.

There are a coordinator unit and multiple terminal nodes in the communication network. The coordinator unit has a broadcast function to multiple radio terminal node scheduling instructions from the PC control software. The terminal node can receive the information transferred from the AGV control system, and routing function can receive the data forwarding, enlarging the coverage of the coordinator unit of instruction [20-22]. The wireless transmission structure is shown in Figure 8.

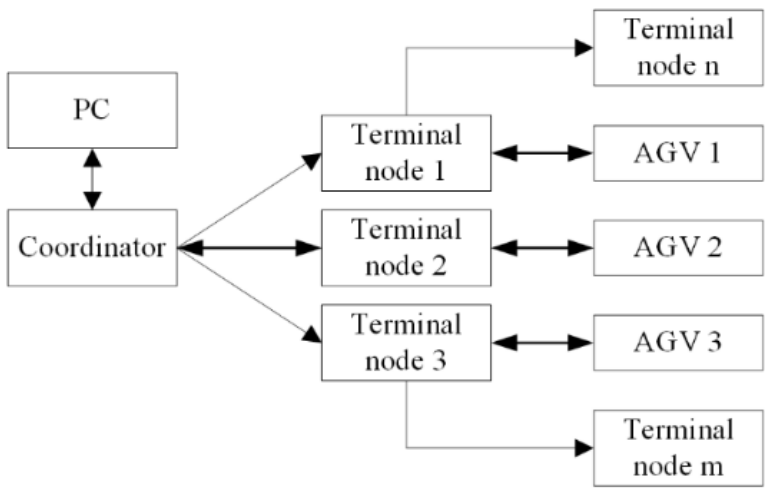

Figure 8 . Wireless transmission structure 


\subsection{Scheduling Flow}

During the operation process of the AGV formation, it relies mainly on the AGV's tracking control to complete the control. The PC control software can control other AGVs to start or stop based on the special work condition of the AGV to achieve the internal multiple AGV system's start or stop. The control process of the PC control software is shown in Figure 9.

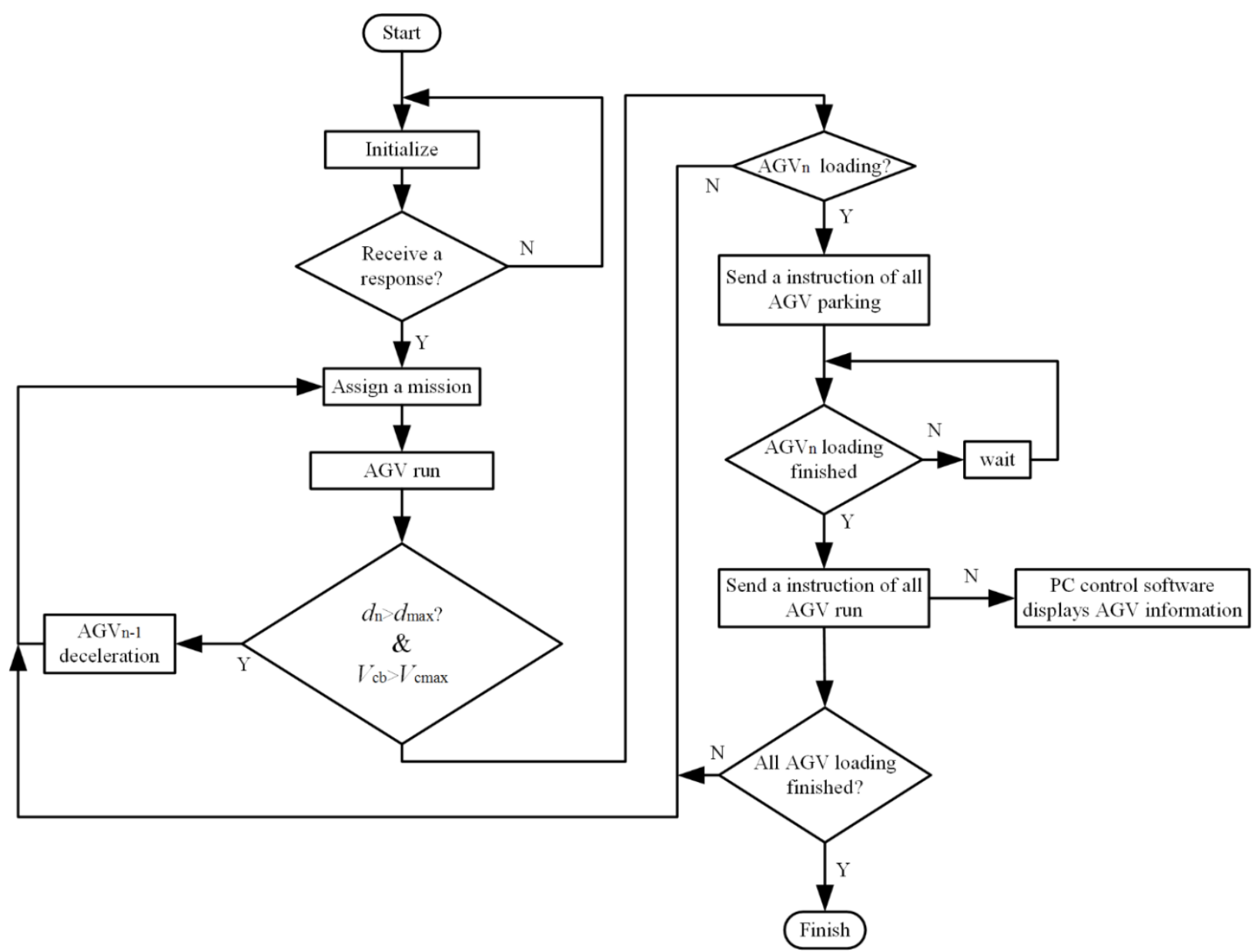

Figure 9. Fleet scheduling process

After the operation information of the AGV is transmitted to the computer, the PC control software filters and classifies the valid information, a part of the valid information is displayed on the screen, and the other part is processed by the software and output as the control signal. The proceed manual control signal is also output as a control signal. The software workflow is shown in Figure 10.

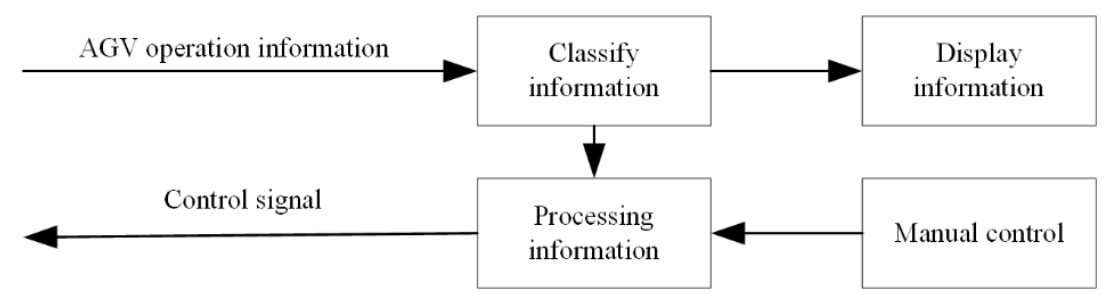

Figure 10. The PC control software workflow

\subsection{Design of the Software interface}

We designed the control software of the PC, shown in Figure 11. Its main interface contains two parts: monitoring and control. This will help us to understand the operation of the system intuitively. The rest of the automatic control work depends on the software within the program to realize the functional requirements. 


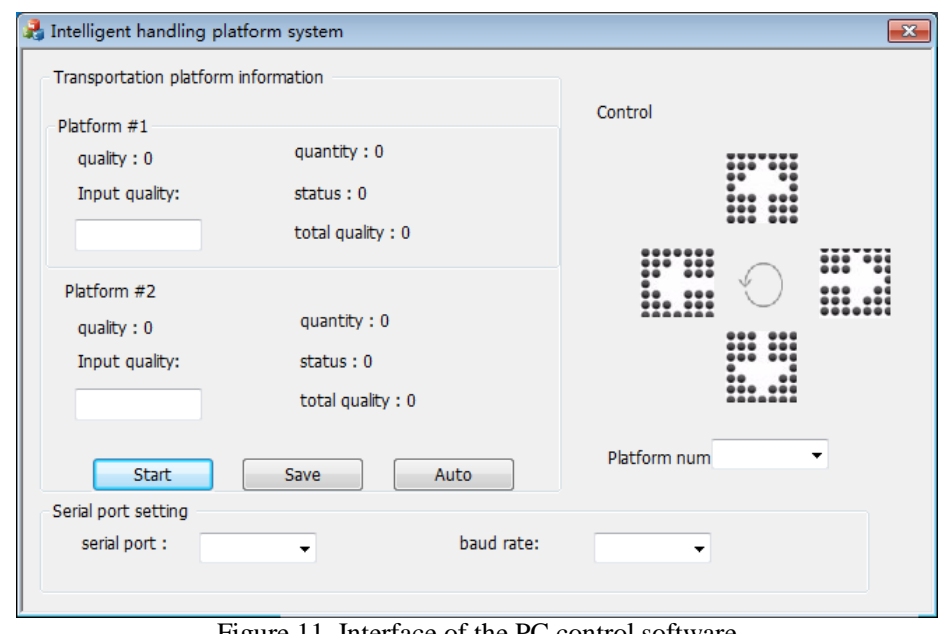

Figure 11. Interface of the PC control software

\section{Experimental Test}

A simple AGV physical model is shown in Figure 12. This model is used to verify the control strategy. Experiments of the physical model and the virtual simulation are carried out below.

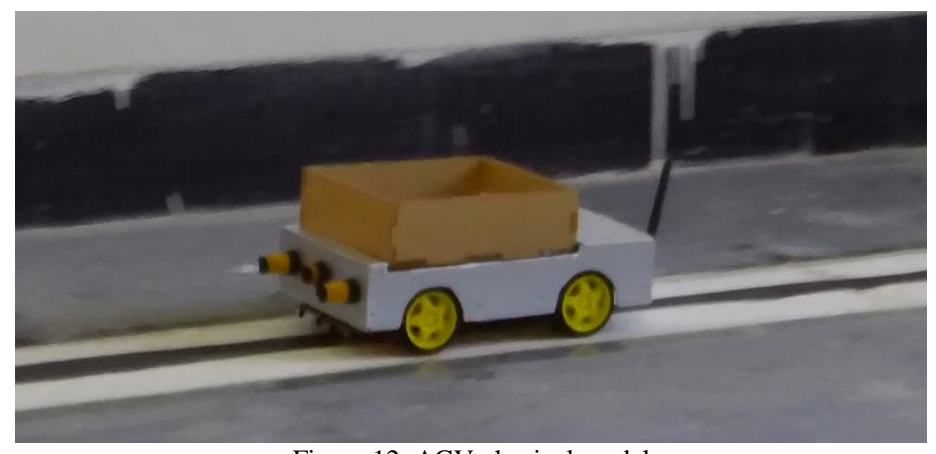

Figure 12. AGV physical model

\subsection{Test of Tracking Accuracy}

During the simulation, the disturbance signal (change deviation $e$ ) is added to the fuzzy controller. The output of the controller can eliminate the error in a relatively short time. Compared with the proportional controller system, this controller has the character of less overshoot and quick response. The position deviation changing with the time is shown in Figure 13.

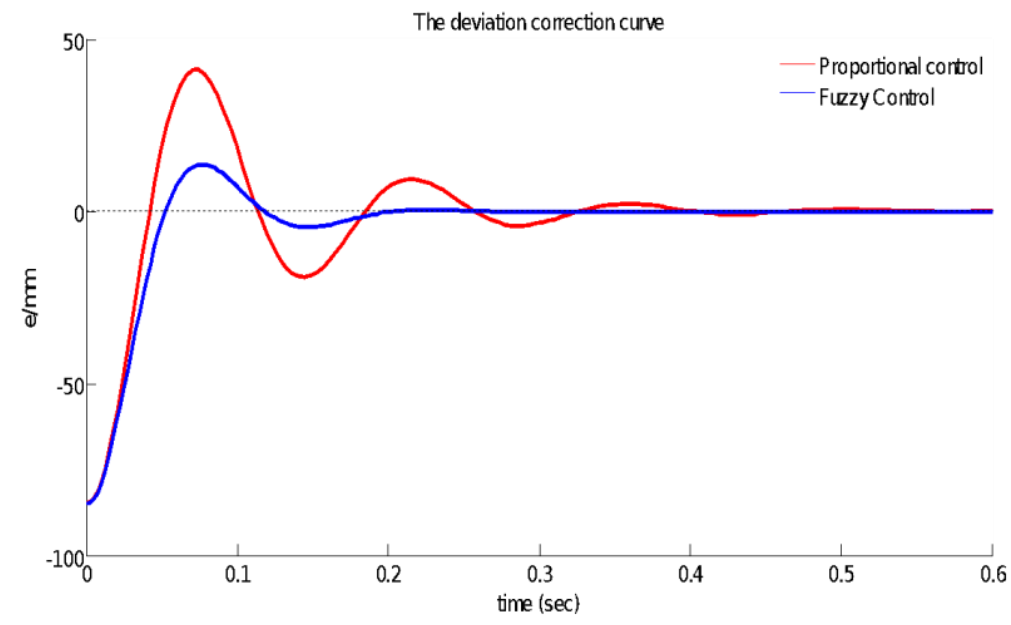

Figure 13. Deviation of the tracking 
To actually carry out the test of the AGV, the test track is represented as a black solid line in Figure 14. The track is composed of multiple line segments and arc closed orbits. This track contains the common straight, intersection and circular arc. Place the dropping ink device in the center of the test vehicle bottom and leave the running trajectory after running. After multiple trajectory fittings, the red dotted line shown in Figure 14 can be obtained. This line can be seen as the actual vehicle trajectory. Compared with the orbit, we can easily find that the whole trajectory is smooth, and it is consistent with the pre-set orbit [23-24].

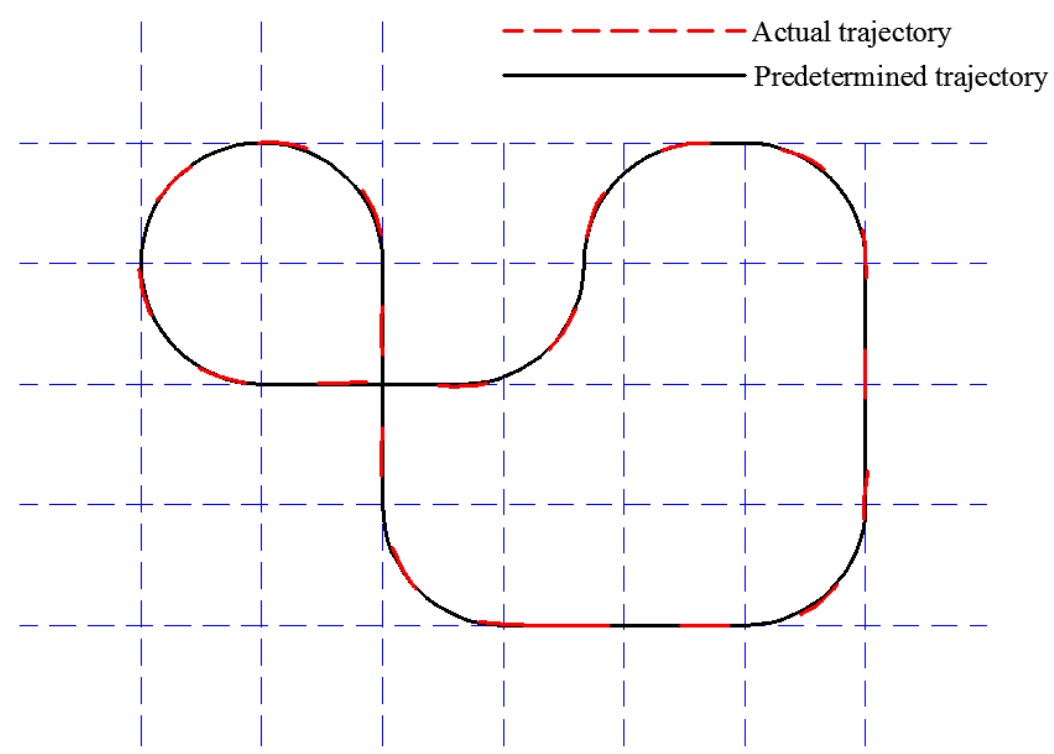

Figure 14. Tracking deviation

\subsection{Test of Following Accuracy}

The simulation test of the tracking control is carried out in MATLAB software. We just set the distance of the front and rear vehicle and added the speed interference factors to achieve the speed curve and the distance between two as shown in Figure 15.
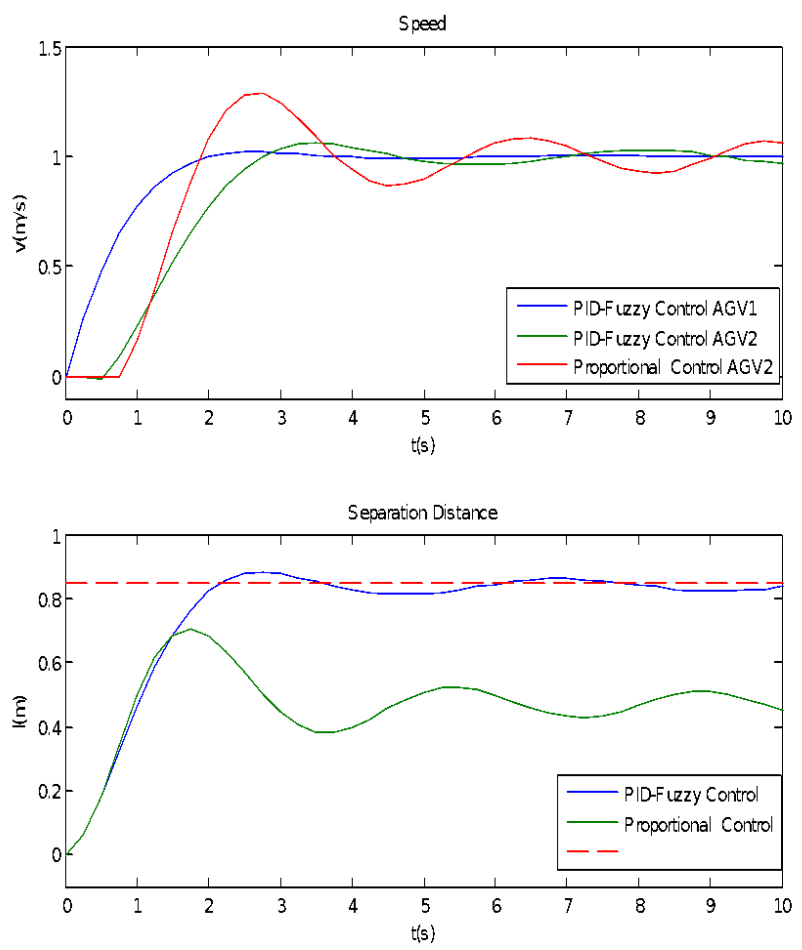

Figure 15. Curve of speed and separation distance 
When adopting the PID-Fuzzy controller, the AGV started in a relatively short period of time with a gentle acceleration process. During this process, there is no large fluctuation in speed, and the distance between the two AGVs remains at a stable value. If only using the proportional controller, the speed fluctuation of the AGV is large and the distance between the two vehicles has a bigger distance than the set value. For the above reasons, the PID-Fuzzy controller has a good effect in the tracking control.

\subsection{Test of Formation Efficient}

The AGV working hours testing is carried on in the total of a $10 \mathrm{~m}$ test experimental orbit. The test scenario is shown in Figure 16.

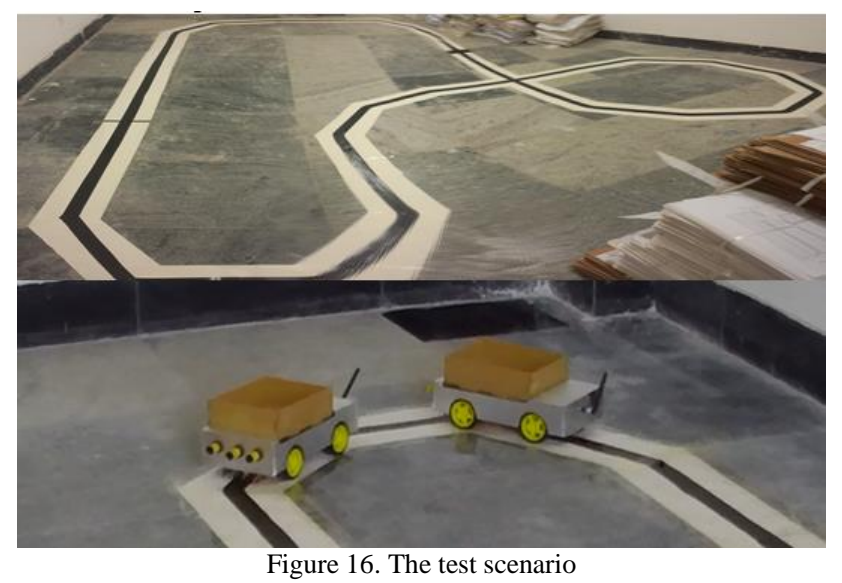

The AGV speed is 0.5 meters per second and the time interval between the stop loading and the restart time is $10 \mathrm{~s}$. We use a single AGV, multiple AGV formation, and multiple AGV non-formation in operation. The working time is recorded in Table 2.

\begin{tabular}{|c|c|c|c|c|}
\hline AGV number & Working mode & Running time (s) & Loading time (s) & Total time (s) \\
\hline Single & Single & 24 & 11 & 35 \\
\hline Five & Multiple & 33 & 53 & 86 \\
\hline Five & Single & 116 & 51 & 167 \\
\hline
\end{tabular}

By the statistics, you can see that the total running time is slightly increased and the loading time is increased with the multiple AGV formation compared with the single AGV. Compared with multiple AGVs running separately in turn, the running time is greatly reduced. Under the condition of reaching to the same capacity, the total time is shortened by half.

From the above tests, it can be concluded that the control strategy designed has better performance and control effect compared with the traditional controller. Additionally, the efficiency of the AGV formation with the PC control software has been improved as well.

\section{Conclusions}

1) In order to solve the tracking and following control questions existing in the AGV automatically control, this paper puts forward the fuzzy control and PID control respectively, and combines these two methods to constitute the Fuzzy-PID hybrid controller to control the running direction and velocity of the AGV.

2)This paper puts forward the AGV formation collaboration method and designs the PC control software to schedule the AGV formation process.

3) The test results show that the hybrid controller can guarantee the stability of the AGV running and following accuracy. Introduction of the PC controller can increase the efficiency of the AGV formation work to verify that this control strategy is effective.

4)The control strategy and the AGV formation collaboration method can be applicated in some similar industrial environments. 


\section{Acknowledgements}

This work is supported by A Project of Shandong Province Higher Educational Science and Technology Program No. J16LB59. The authors thank the anonymous reviewers for their helpful suggestions.

\section{References}

1. H. Xu, Q. Liu, F. Che, and M. Yuan, "Application of Transfer AGV in Materials Transportation System," Logistics Sci-Tech, No. 6, pp. 80-82, 2013

2. Q. P. Wu, Y. P. Jin, P. Ren, and Z. Y. Zha, "Present Situation and Developing Trend of AGV Key Technology," Manufacturing Automation, Vol. 35, No. 5, pp. 106-109+121, 2013

3. J. H. Zhang and Q. S. Gao, "Complicated Logistics System Engineering," Journal of Qingdao University, Vol. 15, No. 2, pp. 75-77, 2002

4. Q. Fan, "Panning and Designing of Automotive Manufacturing Corporation's Distribution Center," Shang Hai Jiao Tong University, pp. 1-5, 2008

5. J. R. Zhang and Y. Ge, "Design of Small-Scare Automatic Handling System," Industry and Mine Automation, No. 5, pp. 105106,2012

6. Z. Huang, "Research on the AGV Technology based on PLC Control and its Application," East China University of Science and Technology, pp. 4-8, 2013

7. C. B. X. Zhang and Z. Q. Huang, "Evolution Summarization of Automated Guided Vehicles (AGV)," Manufacture Information Engineering of China, No. 1, pp. 53-59, 2010

8. Y. Y. Zhang and G. Q. Wang, "Analysis on Influence of Material Handling System in Military Logistics," Department of Material Handling Machineries, Military Traffic Institute, No. 3, pp. 78-79, 2005

9. C. L. Wang, N. Yuan, and X. L. Wang, "Design of the Materials Loading and Handing System at Distribution Centers," Logistics Sci-Tech, No. 5, pp. 67-68, 2011

10. M. S. Jie and W. H. Choi, "Type-2 Fuzzy PID Controller Design for Mobile Robot," International Journal of Control and Automation, Vol. 9, No. 11, pp. 203-214, 2016

11. X. Q. Lian, "Fuzzy Control Technology," China Electric Power Press, pp. 38-42, Beijing, 2003

12. Z. Y. Wang, Y. D. Zhan, and L. M. Zhang, "Fuzzy Control of Two-Wheel Difference Speed Steering in Automated Guided Vehicles based on Vision Computer Applications," Computer Applications, No. 7, pp. 1789-1791, 2007

13. Z. Y. Zhang, J. F. Zhang, L. W. Huang, and C. Y. Li, "Trajectory Tracking Fuzzy Control Algorithm for Picking Robot Arm," International Journal of Control and Automation, Vol. 7, No. 9, pp. 411-422, 2014

14. J. Duan, "Research on Intelligent PID Control Theory and Technique of Digital Electronic Governing System of Locomotive Diesel Engine," Dalian University of Technology, pp. 1-7, 2000

15. Y. H. Lee and H. S. Kim, "Digital Controller Design to Control the Direct Current Motor System," International Journal of Control and Automation, Vol. 7, No. 9, pp. 283-288, 2014

16. A. Husain and R. Abha, "A Hybrid of Sliding Mode Control and Fuzzy Gain Scheduling PID Control using Fuzzy Supervisory Switched System for DC Motor Speed Control System," International Journal of Grid and Distributed Computing, Vol. 9, No. 5, pp. 41-54, 2016

17. Y. Zuo, "Research on the Techniques in Industrial Wireless Mesh Networks," East China University of Science and Technology, pp. 17-39, 2013

18. J. T. Cui and H. Li, "The Remote Data Monitoring Design based on Wireless Sensor Network," International Journal of Future Generation Communication and Networking, Vol. 9, No. 8, pp. 1-8, 2016

19. W. J. Dong, L. H. Li, W. Y. Guo, and H. G. Tang, "Design of the Granary Temperature and Humidity Monitoring System based on Zigbee," Journal of Harbin University of Science and Technology, No. 19, pp. 121-123, 2014

20. Y. Lin, X. Zhu, Z. Zheng, et al., "The Individual Identification Method of Wireless Device based on Dimensionality Reduction and Machine Learning," Journal of Supercomputing, No. 5, pp. 1-18, 2017

21. Y. Lin, C. Wang, J. X. Wang, and Z. Dou, "A Novel Dynamic Spectrum Access Framework based on Reinforcement Learning for Cognitive Radio Sensor Networks," Sensors, Vol. 16, No. 10, pp. 1-22, 2016

22. Y. Lin, C. Wang, C. Ma, et al., "A New Combination Method for Multisensor Conflict Information," Journal of Supercomputing, Vol. 72, No. 7, pp. 2874-2890, 2016

23. Q. Wu, Y. Li, and Y. Lin, "The Application of Nonlocal Total Variation in Image Denoising for Mobile Transmission," Multimedia Tools \& Applications, Vol. 76, No. 16, pp. 1-13, 2016

24. C. Shi, Z. Dou, Y. Lin, et al., "Dynamic Threshold-Setting for RF-Powered Cognitive Radio Networks in Non-Gaussian Noise," Physical Communication, Vol. 27, No. 4, pp. 99-105, 2018 